\title{
Cold Steel Dissection in Phonosurgery: How I Do It
}

\author{
Alok Rathi ${ }^{1}$, Declan Costello ${ }^{2}$
}

\begin{abstract}
Phonomicrosurgery places specific demands on the otolaryngologist. One of the key skills requiring mastery in phonosurgery is cold steel dissection. Here we describe the technique of holding microsurgical instruments with further stability for fine control surgery.

Keywords: Cold steel, Dissection technique, Phonosurgery.

International Journal of Phonosurgery \& Laryngology (2020): 10.5005/jp-journals-10023-1179
\end{abstract}

\section{INTRODUCTION}

Phonomicrosurgery places specific demands on the otolaryngologist. The requirement for fine skills and dexterity means that there is a learning curve which requires patience to overcome. One of the key skills requiring mastery in phonosurgery is cold steel dissection. One can cut short this long learning curve by holding the instrument in the following way.

\section{TECHNIQUe}

Phonosurgical instruments used for cutting/grasping have a proximal mechanism that requires a thumb and a finger through the two holes. However, holding the device with thumb and finger does not allow good control and one need to support it with another finger (Fig. 1A). Furthermore, if the fingers are used in this way, rotation or movement of the instrument requires movement of the whole arm; this can result in unstable or sudden movements of the distal instrument. Significantly, when using two instruments in a narrow laryngoscope, the fingers and thumbs of each hand can impede each other's movements (Fig. 2A).

Holding the instruments in the way suggested-thumb and index finger holding one arm and two fingers holding another arm (Fig. 1B) - allows for more precise control. An additional advantage of using the technique suggested is that control of both the arms can be achieved separately rather than one being dependent on other (Fig. 1B compared with Fig. 1A).

Advantages of holding instruments in this method:

- Excellent control of the instrument by the surgeon.

- Easy maneuverability and rotation while operating on the medial edge of the vocal fold, when compared with the traditional style (Fig. 2B).

- The fingers of each hand do not obstruct each other.

- It is easy to rotate the instrument without needing the operator move their whole arm (Fig. 2B compared with Fig. 1B).

\section{Limitations}

- This technique works only in instruments with the proximal junction box and is difficult with the distal junction box (Fig. 1B).

- This technique is recommended only when finest control of the device is required and one may find the traditional way of holding the device superior when operating on gross laryngeal lesions, e.g., taking a biopsy. \begin{tabular}{l}
\hline \hline 'Department of ENT, Queen Elizabeth Hospital, Birmingham, West \\
Midlands, UK \\
2Department of ENT, Wexham Park Hospital, Slough, Berkshire, UK \\
Corresponding Author: Alok Rathi, Department of ENT, Queen \\
Elizabeth Hospital, Birmingham, West Midlands, UK, Phone: +44 121 \\
6272000, e-mail: alokrathi14@gmail.com \\
How to cite this article: Rathi A, Costello D. Cold Steel Dissection in \\
Phonosurgery: How I Do It. Int J Phonosurg Laryngol 2020;10(1): \\
23-24. \\
Source of support: Nil \\
Conflict of interest: None \\
\hline \hline
\end{tabular}

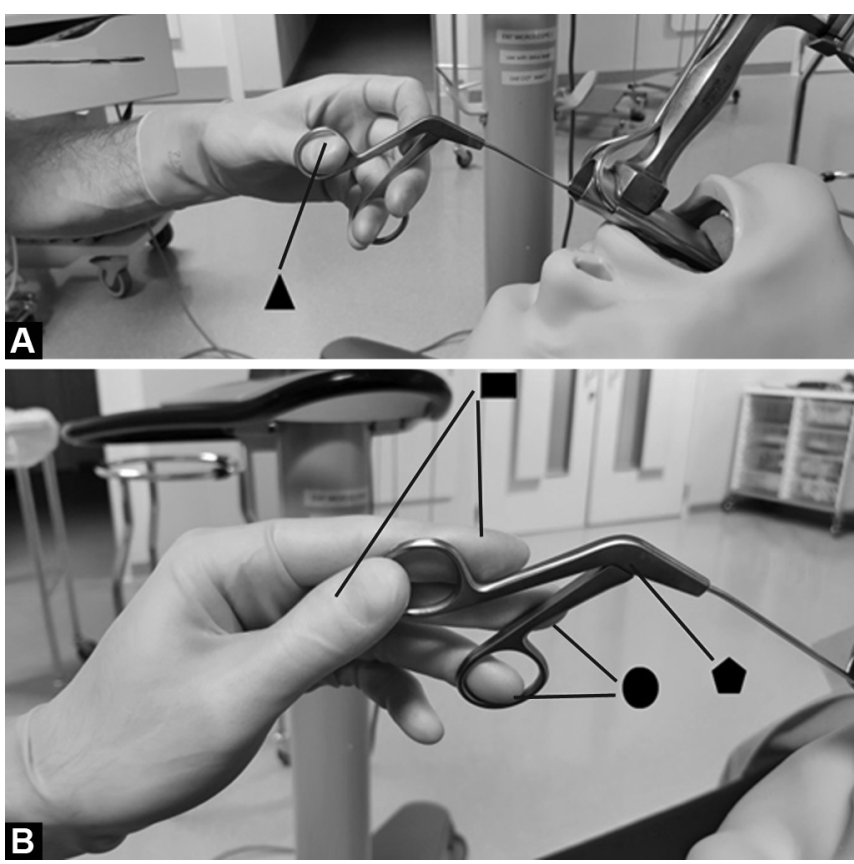

Figs 1A and B: (A) Traditional method of holding instrument. (B) Suggested alternative technique. - Two fingers (thumb and index) holding one arm. - Two fingers holding the other arm. $\Delta$ Only thumb is supporting this arm and it also provides countertraction to other arm. Proximal junction box with mobile proximal arm against fixed distal arm

(0) The Author(s). 2020 Open Access This article is distributed under the terms of the Creative Commons Attribution 4.0 International License (https://creativecommons. org/licenses/by-nc/4.0/), which permits unrestricted use, distribution, and non-commercial reproduction in any medium, provided you give appropriate credit to the original author(s) and the source, provide a link to the Creative Commons license, and indicate if changes were made. The Creative Commons Public Domain Dedication waiver (http://creativecommons.org/publicdomain/zero/1.0/) applies to the data made available in this article, unless otherwise stated. 

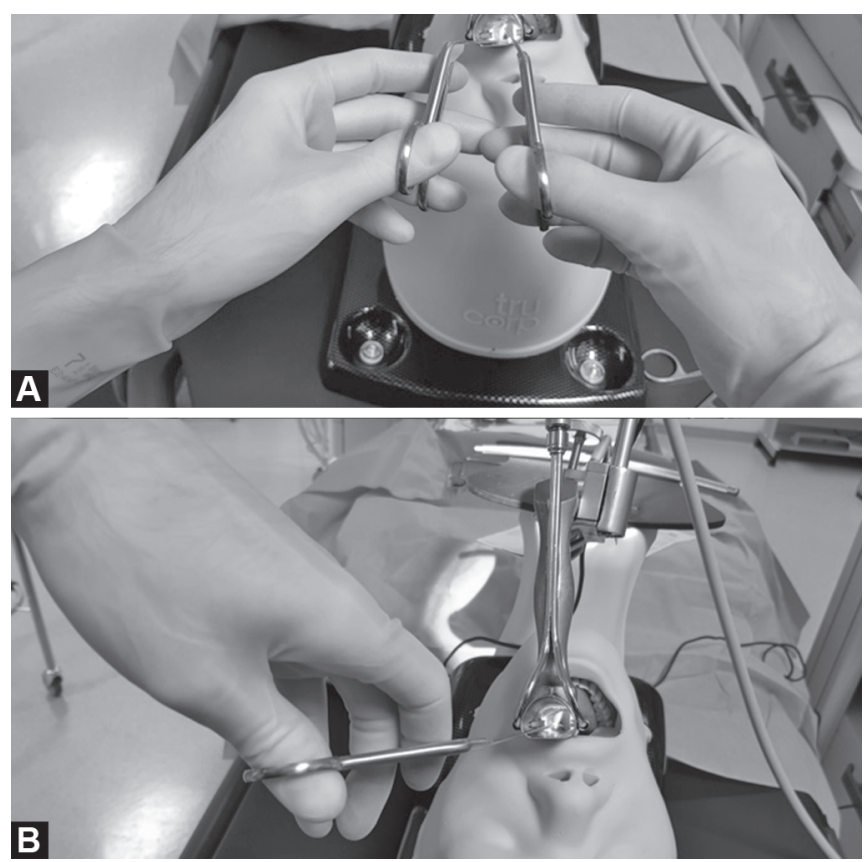

Figs $2 A$ and $B$ : (A) Using the traditional technique, fingers of opposing hands can restrict movement. (B) When holding the instrument in a traditional way, rotation of the instrument relies on movement of the whole of the operator's arm, resulting in loss of control

\section{Discussion}

The literature thus far has always focused on techniques of surgical dissection and less on the means of holding the instruments. It is well recognized that phonomicrosurgical instruments are difficult to manipulate because of their length, because the fulcrum effect of the instrument (resting proximally on the laryngoscope) amplifies movement of the tip of the instrument distally.

The trainee otolaryngologist will often have an opportunity to excise relatively straightforward lesions such as polyps on the free edge of the vocal fold. One of the key skills to master is in controlling the movement of the tips of the instruments (whether grasping forceps, dissectors, blades, or scissors). We have previously described a technique for improving stability by resting the elbows. ${ }^{1}$ We now describe a technique for holding the microsurgical instruments, which gives further stability for fine-control vocal fold surgery.

\section{Conclusion}

Phonomicrosurgery is technically demanding. There is a learning curve to acquire the fine skills and dexterity. Holding phonomicrosurgery instruments in the described technique is helpful. Stability and fine control at the tip of the instruments is easily achieved.

\section{Ethical Approval}

For this type of study, formal consent is not required. All procedures performed in this study were in accordance with the ethical standards of the National Research Committee and with the 1964 Helsinki Declaration and its later amendments.

\section{Reference}

1. Osborne MS, Mitchell-Innes A, Costello D. A helpful technique for manual stability and avoiding fatigue during microlaryngoscopy. Clin Otolaryngol 2015;40(2):169-170. DOI: 10.1111/coa.12292. 